\title{
Perceptions and experiences of caregivers of severely malnourished children receiving inpatient care in
} Malawi: An exploratory study

Selena Gleadow Ware ${ }^{1}$, Allison I Daniel ${ }^{2}$, Chiwoza Bandawe ${ }^{3}$, Yohane Paulo Mulaheya ${ }^{4}$, Sylvester Nkunika ${ }^{4}$, Dumisani Nkhoma ${ }^{4}$, Demoubly Kokota $^{5}$, Robert C Stewart ${ }^{6}$, Wieger Voskuij ${ }^{7}$

\author{
1. Global Mental Health Academic Network, University of Edinburgh; Department of Mental Health, \\ College of Medicine, University of Malawi \\ 2. Centre for Global Child Health, Hospital for Sick Children, Toronto Ontario, Canada; Department of \\ Nutritional Sciences, University of Toronto Faculty of Medicine, Toronto, Ontario, Canada \\ 3. Department of Mental Health, College of Medicine, University of Malawi \\ 4. Queen Elizabeth Central Hospital, Malawi \\ 5. Department of Paediatrics and Child Health, College of Medicine, University of Malawi \\ 6. Division of Psychiatry, University of Edinburgh; Department of Mental Health, College of Medicine, \\ University of Malawi \\ 7. Department of Paediatrics and Child Health, College of Medicine, University of Malawi; Global Child \\ Health Group, Emma Children's Hospital, Academic Medical Center, Amsterdam, The Netherlands
}

\begin{abstract}
Background
Severe acute malnutrition (SAM) affects approximately 18 million children under the age of five and is associated with more than 500 thousand deaths per year. Existing research has indicated that a high number of caregivers of children admitted for inpatient treatment of SAM experience psychological distress, depressive symptoms, and suicidality. However, no published studies in Malawi have been undertaken to qualitatively explore caregivers' perceptions and experiences regarding their children's malnutrition and inpatient treatment.

Aim

To explore caregivers' perceptions and experiences surrounding childhood acute malnutrition and the experience of inpatient care in Malawi.

Methods

Interviews were conducted utilising a semi-structured topic guide and were coded using thematic analysis.

Results

Caregivers $(\mathrm{N}=30)$ gave informed consent to participate in interviews. Caregiver understanding and perceptions of their children's illness varied. Some caregivers identified a physical cause, with a minority identifying lack of dietary protein. Other narratives were around characteristics of the infant, other circumstantial events and religious and spiritual influences. One-third of caregivers described their own health difficulties and marital and relationship stressors. Challenges such as poverty, lack of access to food, poor food variability and competing demands for caregiver time were explained. Both positive and negative experiences of family and community support and hospital-based care were reported.

Conclusion

The themes identified contribute to a greater contextual understanding of the multifactorial and integrated approaches required to address malnutrition. This study indicates that healthcare providers need to take a multi-faceted view of malnutrition and be aware of the many factors that may influence healthcare experience and response to treatment. Acknowledging pluralistic belief systems may improve engagement with care. This demands a broader appreciation of perceptions and experiences of malnutrition, hospitalbased care, sources of support and stressors within the caregiver, family and community environment as well as consideration of social determinants such as poverty and how these influences present within a clinical context.
\end{abstract}

Key words: maternal mental health, nutritional rehabilitation unit, severe malnutrition, caregiver experience

\section{Introduction}

Almost 6 million deaths occur globally each year in children under five ${ }^{1}$. Malnutrition is directly or indirectly responsible for approximately $45 \%$ of these deaths, with mechanisms including fetal growth restriction, suboptimal breastfeeding, stunting, wasting, and inadequate micronutrient supply ${ }^{1-3}$. In Malawi, a high proportion of children under five are affected by malnutrition, with $12 \%, 37 \%$ and $3 \%$ being underweight, stunted, and wasted respectively ${ }^{4}$.

Severe acute malnutrition (SAM) is diagnosed in children with at least one of the following: a mid-upper-arm circumference below $11.5 \mathrm{~cm}$ (severe wasting), a weight-forlength or -height z-score less than -3 SD below the median (severe wasting), and/or the presence of bilateral pitting oedema (kwashiorkor) ${ }^{5}$. If medical complications develop, such as systemic infection, HIV disease, or respiratory infection, inpatient treatment is required ${ }^{5-7}$. Approximately 600 severely malnourished children are admitted each year to the Moyo Nutritional Rehabilitation and Research Unit at the Queen Elizabeth Central Hospital in Blantyre, Malawi, a tertiary referral site $^{8}$. During hospitalization, primary caregivers are responsible for feeding their child every 
three hours, ${ }^{6}$. This is a highly demanding role that requires throughout the day and night.

A prospective study of psychological distress amonost mothers of children admitted to a nutritional rehabilitation unit in Malawi identified that $71 \%$ of mothers scored significantly high on a screening tool for depression, the Self-Reporting Questionnaire (SRQ-20), and that $15.6 \%$ reported experiencing suicidal ideation in the previous fou levels of depression included having an older child, lack of confiding relationship with a spouse, and having a previous child die

Researchers have clearly established an association between maternal mental health and child growth and development. Several different pathways link these domains and are thought to operate bi-directionally 10,11 . In Malawi, community-based research has indicated that up to $30 \%$ of mothers attending an under-five immunization clinic scored positively for depression ${ }^{12}$. This was sionificantly associated with reduced lenoth-for-age in their children ${ }^{12}$ Evidence from highincome countries has also indicated that post-natal depressive symptoms are inversely associated with responsive feeding of infants ${ }^{1}$

The need for interventions to take into consideration the complex contextual factors that are associated with childhood malnutrition has been recognized, particularly within an integrated early childhood development framework ${ }^{14}$ Whilst it is known that nutrition-specific interventions are necessary, it is acknowledged that they are not sufficient ${ }^{14,1}$. Interventions such as behaviour change communication and responsive parenting have provided promising benefits. However, there has been limited exploration of underlying cultural barriers that may affect feeding practices, and the Qualitative interviews have not been conlucted on the Qupulation of inpatient caregivers of infants admited for SAM. It is not clear if this group varies significantly from community samples of infants and their families examing infant feeding, compared to management of SAM.

Thus, while targeting psychosocial influences have been dentified as a potential area of intervention for both

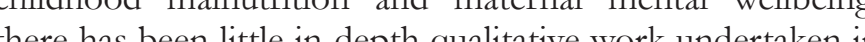
low- and middle-income countries to explore experiences f are between the known influences on chilhood malnutrition and maternal mental health and wellbeing Using a qualitative phe the and experiences of car SAM within a

\section{Methods}

Study design

This study was qualitative, aiming to obtain information regarding the psychological and social context of caregivers and their children which may not be identified during the use of routine questionnaires or clinical care. A qualitative metho a caregivers, who play an important role in recovery from during routine care and understanding the perspectives of caregivers is important if longer term outcomes are to be ingroved. Semi-structured interviews were chosen to allow chers to disclose thoughts and feelings that they might not feel comfortable disclosing within a group environment, particularly as psychosocial stressors and mental health are to enable an open framework of discussion, allowing the interviewer and participant to follow a structure conversation yet also explore topics in greater detail.

\section{Participants}

The study was conducted at the Moyo Nutritional Rehabilitation and Research Unit of the Paediatric Department at the Queen Elizabeth Central Hospital Blantyre, Malawi.

The inclusion criteria were caregivers of children between 6 and 59 months admitted to Moyo for inpatient treatment of SAM defined by the WHO criteria as:

1) Weight-for-length or -height z-scores below -3 SD from the median;

Mid-upper-arm circumference of below $11.5 \mathrm{~cm}$; and $/$ or 3) Nutritionally-induced bilateral pitting oedema 5 .

This age range corresponded with the age range of children admitted to the SAM ward at the time of the study.

Caregivers included the primary caregivers or guardians for the infant during their hospital stay, often the child's mother but not exclusively. In Malawi, each child admitted to hospital is accompanied by a guardian who may be a mother, close family menber or other caregiver. In this study, the during hopital a purposive sampling and participants were entolled after provosive sampling and participants were enrolled after proving infor con earegivers who were not willing or able to provide informe

\section{Data collection}

Data collection occurred between 31 March and 2 May 2014 with recruitment of 30 caregivers. Baseline demographic level, residential secting occupation age, parity, educationa structured interviews were conducted in the local language (Chichewa) by three male Malawian medical students and were audio-recorded. A topic guide was developed by identifying themes from the existing literature and consulting clinicians involved in management of SAM and mental health. It was used as a basis for the interview and encouraged discussion on the following topics: child health and wellbeing, caregiver wellbeing and concerns, food security, social support and the experience of hospital admission.

\section{Ethical consideration}

This study was approved by the College of Medicin Research Ethics Committee (SP 061317). Verbal and written information was provided (in Chichewa or English). Written consent was obtained; for participants unable to read or write, the information was read in Chichewa and consent by signature or thumbprint was witnessed.

For caregivers who were identified as having ongoing mental ealth problems or social difficulties, a referral pathway for assessment at the local psychiatric clinic was avallable.

Data management and analysis

Interviews were anonymized and transcribed verbatim from

perceptions and attributions of infant health and illness; cross-checked the back-translation of the interviews for maternal wellbeing and interpersonal relationship stressors; accuracy. Data were coded using thematic analysis by two family and community supports and stressors; and household reviewers: one research assistant, and one supervisor of the resource limitations such as food insecurity, unemployment, project $(\mathrm{SGW})$. Coding was structured around the key topics poor housing, and material conditions of poverty. Positive of the interview and emerging themes. Both researchers and negative experiences of hospital-based care were also reviewed and coded the manuscripts independently, before reviewing areas of difference together and agreeing final themes. The final themes were cross-checked with one of the senior researchers $(\mathrm{CB})$. Interview data were reported descriptively and illustrative quotes were used anonymously where appropriate. Contextual information that could identify the participants was removed.

\section{Results}

\section{Baseline demographics of participants}

Of 30 caregivers enrolled, interview data was available for analysis on 27 participants, as three of the interview audio files were corrupted and not possible to analyse. Characteristics and demographic data of caregivers and their children are also the mother of the infant, a minority were another family member or social contact such as neighbor $(\mathrm{n}=3)$.

Table 1: Characteristics

\section{Child characteristics}

\begin{tabular}{ll}
\hline Age (months), median (range) & $24.0(9-48)$ \\
\hline Sex, $n(\%)$ & \\
\hline Male & $11(37)$ \\
\hline Female & $19(63)$
\end{tabular}

Female

Caregiver characteristics and demographics

Age (years), median (range) 24.5 (16-36)

Education, $\mathrm{n}(\%)$

Primary

Secondary

Living setting, $\mathrm{n}(\%)$

Rural

Peri-urban

Urban

\section{Maternal Occupation, $\mathrm{n}(\%)$}

Business

Cleaner

Housewife

Student

Farmer

HIV status, $n(\%)$

Positive

Negative

Unknown

Maternal Marital Status, $\mathrm{n}(\%)$

Single

Married

Divorced/ Widowed

Core themes

Recurrent themes identified from the interviews included

\section{discussed by caregivers.}

Percep

Caregiver understanding and perceptions of their children's illnesses varied. In some descriptions ( $\mathrm{n}=5)$, the children's illnesses were understood in terms of a physical cause. Several informants gave a narrative of seeking help for a particular physical health presentation, such as diarrhoea or malaria which was later narrated as being related to malnutrition. The undestanding of malntition as the cause was not universal in these narratives, with some caregivers remaining

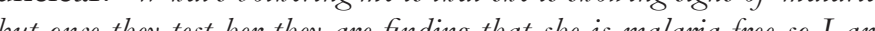

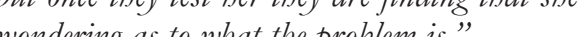

In some instances, caregivers described features of clinical presentation of malnutrition, but did not indicate specifically that this was malnutrition, rather describing other potentia causes such as 'lack of blood'. Whilst some caregivers demonstrated an understanding of inadequate nutritional intake as a cause of the child's illness, specific nutrients such as proten were not mentioned. Other food groups were highlighted such as a lack of fruit and vegetables. Caregivers identified that the lack of nutritional intake did not necessarily relate to intake of nsima (a form of maize), the different influencing factors were identified, which include different influencing factors were identified, which included social triggers and circumstantial events. Some of the physical features of malnutition were identified and some understanding of the role of inadequate nutritional intake in

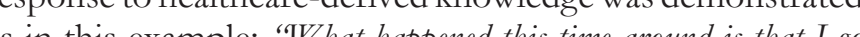
as in this example: "Wat happened this time around is that I got [the healthcare professional] are saying it is because be lacks food in his body... but they don't mean food like nsima but fruits and vegetables." A small number of caregivers ( $\mathrm{n}=3$ ) identified their children as difficult or "fussy" eaters, which affected nutritional intake. In these narratives, the impact of a child being a difficul eater was amplified by the care environment, such as lack of persistence within nursery or school feeding programs to address the specific child's needs, as in this example: "We are keay only that he is a difficult child. I go to school and so does be and when he comes back and they attempt to feed bim, once be refuses the let him be because there are other children in need of care there too." A proportion of caregivers $(n=6)$ identified religious or spiritual influences as alternative causes of childhood ilnesses. At times, these attributions were described when he caregiver did not have a clear medical explanation for the child's illness, as in this example: "He has many health problems every month as it seems he was bewitched. We came bere after be fainted and the doctors were unable to find any sickenss." (Interview 8, 36 years, small-scale business woman, peri-urban setting)

A small number of caregivers related the impact of the illness on their relationship with the child and the extended household environment, highlighting how child illness may have much wider repercussions for caregiver health and household wellbeing: "T was affected in such a way that when a 
child is sick, notbing progresses in the home and he isn't happy when

Maternal wellbeing, resilience and interpersonal relationsbi

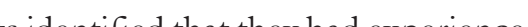
(n) during pregnancy, the postnatal period, or at the time of the during pregnancy, the postnatal period, or at the time of the their current health difficulties originated from pregnancy informants were other caregivers, as the mothers of the children were deceased or unwell. In one case, this of some to relate to a severe mental helth condition of the mother which rendered her unable to care for her child:

"She's [mother of infant] at home. She's got a mental health problem. As soon as she gave birth. Her husband married someone else and so we suspect that the other woman is the one who bewitched her... No, she
doesn't talk. She just goes to drink. water when she is thirsty. We feed her because ber hands no longer function. At night, she doesn't want to sleep but rather to walk around. I have just gotten the news whilst here sleep but rather to walke around. I have just gotten she "

Caregivers described fears and anxieties in relation to a number of marital and relationship stressors. In several cases, there was a clear description of interpersonal events
such as the husband leaving the household, after which food such as the husband leaving the household, after whic
provision and survival became more challenging.

When reflecting on pregnancy and the postnatal period, caregivers described experiencing different levels of support from the main household providers, their husbands or partners.

Relationship difficulties were described by a third of participants $(n=9)$, with a number of different sub-themes emerging including: the father of the child not accepting responsibility or involvement with the caregiver after conception; the husband being unemployed and not providing for the family; relationship difficulties and arguments; the the family home; infidelity; domestic violence; the husband living in a different geographical area; and the impact of substance misuse. In one case, the father of the child was described as being physically unwell due to HIV and unable to support the family. Some informants described beliefs that witchcraft was involved as a cause of some of these difficulties, such as infidelity.

Relationship difficulties were thus described as having direct effect on caregivers' wellbeing and nutritional status, particularly where the informant is dependent on her husband for food security, as is the sociocultural norm, as is not well with my busband. ...We argue and stay for a whole month without talking to each other, sometimes he goes to work, without leaving money to buy food."

However, many caregivers $(\mathrm{n}=13)$ described positive both positive and negat and 4 informants gave descriptions

\section{Family and community supports and stressors}

Family supports and stressors were commonly mentioned by participants. A minority $(\mathrm{n}=5)$ reported difficulties in looking after their children. In some cases, there was regret about having another child and description of child-rearing capacity being overstretched, indicating a compromise of
Perceptions and experience of healthcare access and treatment

Challenges identified by informants included difficulties in accessing appropriate and timely care in the community. rehabilitation unit were reported, including recognition progress, provision of food, and support: "His cheeks would shrink, because he had diarrboea. They told me to feed him Than ORS [Oral Rehydration Salts]. Things wouldn't change and peophe encouraged me to come here. I think things have improved for me here." However, a s all number of informants ingered concen regarding the hospital-based care they were receiving. This it was difficult to clatify from the accounts whether though tributed to the illess or the quality of care recivel limited understanding of hospital treatment protocols: ' $W$ are just warried if our children will come out al. Some pope are just worried if onr childrn will come oun alive. Some people are

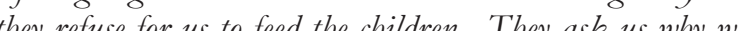
they refuse for us to feed the children. They ask us why we are giving

\section{Discussion}

The findings from this study provide further information regarding the complex social context of SAM and the experiences and perceptions of caregivers of children admitted for inpatient treatment at a nutritional rehabilitation unit. The emerging themes identified are similar to findings from other qualitative studies in Malawi exploring the experience of caregivers in the community in relation to mental distress, notably poverty, lack of support, and fears of witchcraft ${ }^{1}$. Relationship stressors have also previously been identified as stressors to caregivers in the community particularly in relation to fears regarding infidelity, abuse, and bandonment

The experiences described by caregivers illuminate some of the tensions faced particularly when there is reliance on other providers for income and food and where the provider is unable or unwilling to meet these obligations, in the absence of other community supports or social security system. Caregivers may have limited power or choice over food purchasing and decision-making to improve dietary intake of their children. Almost one-fifth of caregivers indicated that they were struggling to meet the demands placed on them

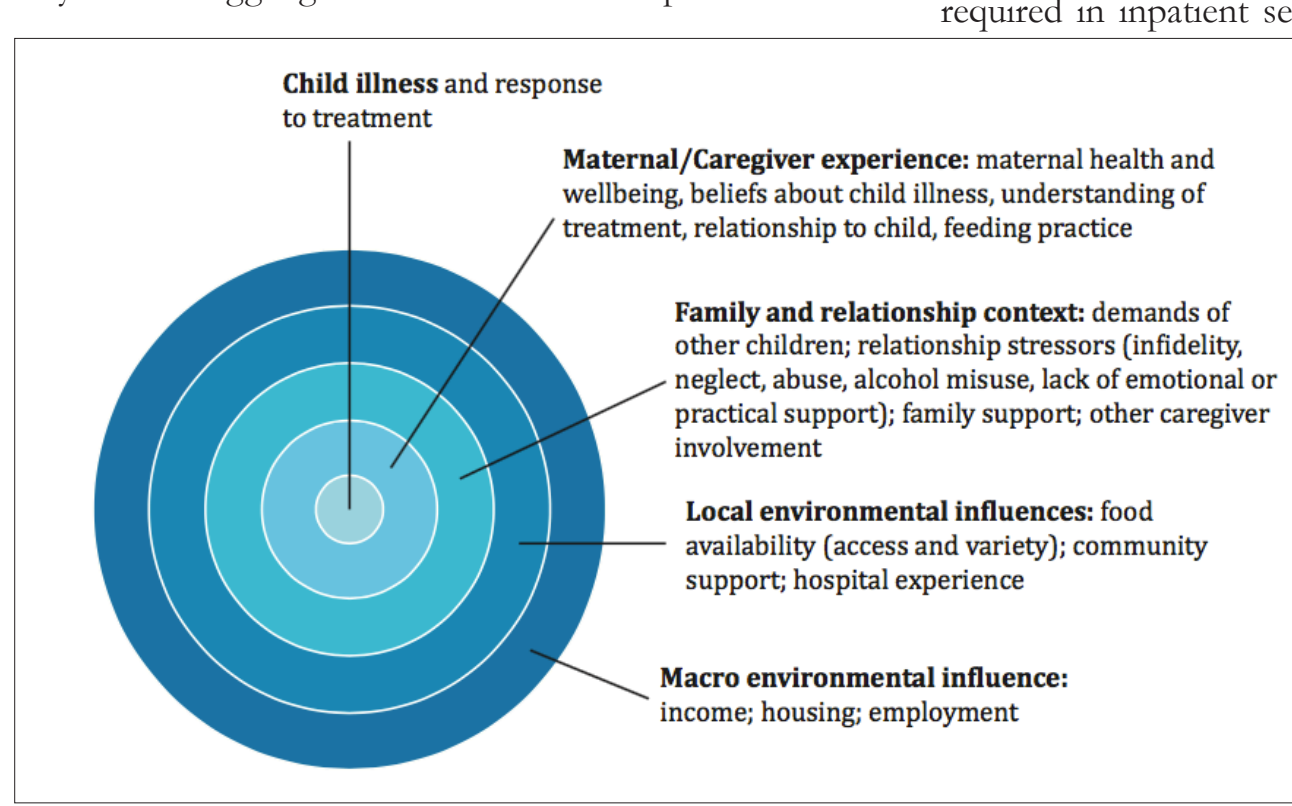

Figure 1: Framework of factors influencing caregivers of children with severe acute malnutritio of their children. However, a small number of informants reported concerns

high birthrate in Malawi and lack of birth spacing In order to care properly for their children and to ensure thentional recovery, they need additional support from healthcare professionals and social care services. This could the identifying if lack of food, limited food diversity or ack of decision-making power is a contributing factor and Anposting families to relevant community-based programs. Additional narratives highlighted situations in which children are cared for by siblings, neighbours, or other care providers requirements and emotional care needs. In these contexts, the mother may not be the main care provider for a child and has little influence or agency over certain aspects of care such as emotional need and attention, which may not be recognized as important by other caregivers. to play in identifying and providing additional social support

The results clearly highlight the vulnerability of caregivers to dependence on the quality of their support network are at play caregivers may have limited capacity to ensors appropriate nutrition care and provision for their families, particularly in this low-income setting where there are very limited or no other forms of financial or material support. Informants also highlighted a number of themes in relation to the understandings and attribution of causality relation to attributions to spiritual and religious influrly in and perosions furthercestors of treatment failure. These factors require provide collo the range of aributions benefit short-term manaement and longer-term recovery for exam by imagement and longer-term recovery, alliance and concordance. Evidence from other countries indicates that if healthcare providers acknowledge pluralistic elief sys explicitly usave of healthcare services can belief systems explicitly, usage of healthcare services can Others reported being able to access a regular supply of food, albeit with limited dietary diversity, but having othe material limitations in the household. Several caregivers a regular basis and the impact this has on the caregiver: "It was very difficult for me, even to find food for me and my children... it is difficult for me to find food to feed them ... g get stressed because I am the only one taking care of them."

Work-related stressors and the challenges of sustaining work while looking after children were also described. For those informants who reported being dependent on farming tensonal availability of food was identified as an issue, highlighting vulnerability to climate change.

A minority of informants $(\mathrm{n}=2)$ described good food availability within the household: "I take maize, fish, beans, groundnuts and grind together. If this flour is not available, we simply
take oil and drop a little to the porridge and then feed it to the child. take oil and drop a little to the porridge and then feed it to the child.
Sometimes we feed him porridge with ground nut flour at seven o.clo at eight o'clock we mash potatoes or pumpkins with sugar and then

Several informants $(\mathrm{n}=3)$ reported poor housing; in som cases, houses had fallen down while the caregiver was
hospital, a potential barrier to community recoverv derstand the intervention being provided. This is particularly important in the context of inpatien schedules at nutritional the feeding units can be demanding especially where a child has poor appetite or other feeding problems. Caregivers may strugole to encourage their children to feed, particularly if their children are acutely ill. Careoivers may also be juggling a variety of competing jughes a variety of children at home and may have the community, and healthcare staff may not be aware of these factors. This study indicates that healthcare providers need to take a multi-
faceted view of malnutrition limited support systems in place in 
and be aware of the multiple factors that may influence which can encourage the development of 'soft' transferable healtheare experience and response to treatment. Healthcare interventions need to be multi-faceted and address the wide family and social contexts, as simply providing nutritiona ehabilitation may not address these underlying complex factors. Using a framework approach (Figure 1) may help clinicians consider a holistic approach to supporting children, their caregivers, and families to recover.

In addition, it may be important to identify subgroups such as vulnerable families that require tailored interventions, including adapting information to improve understanding and collaborative care, and signposting to other community

Commonalities and exceptions in the narratives highlighted other subgtoups warranting further explontion such as those caregivers who have access to reasonable food supply and variety; those caregivers who have positive support but not access to adequate nutrition; caregivers who do not have access to social support; and those who are relinnt on other caregivers within their neighbourhood and family.

This research contributes to the call for formative research to identify local practices and barriers to optimal feeding ${ }^{1 /}$. tt adds to the literature that has indicated the importance of unch as fine undertaken in other low-income countries like Bangladesh ${ }^{1}$

\section{Study limitations}

Limited information was provided by informants regarding the nature of the emotional relationship to the affected child. This may have been due to informants feeling this information was irrelevant, the interview process, or cultur differences in ways of describing the caregiver-infant relationship.

Some informants provided a narrative that appeared to Provide conflicting information such as agreeing that they the interview disclosing a lack of nutritional sources. This may have been due to interviewer bias, performance social acceptance bias, or to the interviewers' limitations in exploring their initial meanings in adequate depth.

Other limitations included that since this study employed purposive and opportunistic sampling, other views may not have been represented. However, a sample size of approximately 30 is typically adequate for data saturation in qualitative sample ${ }^{19}$.

This study only included infants from 6 to 59 months of age. SAM affects infants and children of all ages; therefore, the findings are limited to this age range. Future studies may be valuable to explore the experiences from more specific age
bands across a wider range of years, to ascertain whethe bands across a wider range of years, to ascertain whether
certain experiences are associated with specific age cohorts. The approach of this study, to use medical students to
undertake qualitative research, enabled information to be undertake qualitative research, enabled information to be generated; but the depth of data was limited. Interviews were undertaken by three male medical students. Their own perceptions and different socioeconomic position in relation to the caregivers may have influenced the informatio provided during the interviews and contributed to bias.

The experience of using medical students for interviews and data gathering also highlights the need for medical students to receive undergraduate qualitative method skills training,
.

\section{Conclusions}

In summary, this exploratory qualitative study contributes to the evidence supporting the need to consider the multactorlal nature of ehildhood malnutrition, and and to explicitly engage with

\section{ct of interest statement}

\section{Acknowledgements}

Kate Chidzalo assisted with the transcription and coding of the manuscripts. Dumisani Nkhoma, Yohane Mulaheya nd Sylvester Nkunika undertook interviews of participants. Jacqueline Daniel assisted with graphic production.

None

\section{Author contribution}

$S$ Gleadow Ware and W Voskujl contributed to the design and implementation of the study. Coding was undertaken zalo and S Gleadow Ware and cross-checked (the

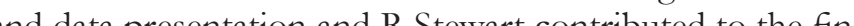

\section{References}

I. Liu L, Oza S, Hogan D, Chu Y, Perin J, Zhu J, et al. Global, regiona and national causes of under-5 mortality in 2000-15: an updated Geals 6736(16)31593-8

2. Black RE, Victora CG, Walker SP, Bhutta ZA, Christian P, De Onis $\mathrm{M}$, et al. Maternal and child undernutrition and overweight in low451. doi:10.1016s0140-6736(13)6037-X'

3. Haddad L, Achadi E, Bendech MA, et al. The Global Nutrition Report

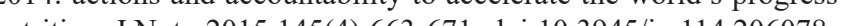

J J International Malawi Zomba, Malawi and Rockville, Maryland; 2016.

5. World Health Organization. WHO Child Growth Standards and the Identification

6. Ashworth A, Khanum S, Jackson A, Schofield C. Guidelines for the Inpatient Treatment of Severely Malnourished Children. (World Healt Organization, ed.). Geneva; 2003

7. World Health Organization. Guideline: Updates on the Managemen .

8. Kouam E. Evaluation of Community Management of Acute

9. Stewart RRC, Bunn J, Vokhiwa M, Umar E, Kauye F, Tomenson B, hildren prospective study of psychological distress among mothers of Care, Heal Dev He nut Dev. 2011;37(1):55-63. do 10.111. Child recent evidence. Matern encourages clinicians to identify the sociocultural, family caregivers' pluralistic belief systems.

No competing interests are declared by the authors.

\section{Source of funding}

Demographic and Heffice (NSO) and ICF Intendicators Report. $22142010.01111 \mathrm{x}$-al Dev. 2011,37(1).55-63. doi:10.111/1.136510. Stewart RC. Maternal depression and infant growth: a review of and illness in Bangladesh, Vietnam and Ethiopia. Public Health Nur. 2014;17(6):1318-1327. doi:10.1017/S1368980013001043

12. Stewart RC, Umar E, Kauye F, Bunn J, Vokhiwa M, Fitzgerald M, common mental disorder and infant growth - a crossectional study from Malawi. Matern Child Nutr. 2008;4(3):209-219. doi:10.1111/j.1740-8709.2008.00147.x

13. Mallan KM, Daniels LA, Wilson JL, Jansen E Nicholson JM Association between maternal depressive symptoms in the early postnatal period and responsiveness in feeding at child age 2 years. Matern Child Nutr. 2015;11(4):926-935. doi:10.1111/men.12116

14. Pérez-Escamilla R, Moran VH. The role of nutrition in integrated early child development in the 21 st century: contribution from 2017;13(1):e12387. doi:10.1111/men.12387

15. Bhutta ZA, Ahmed T, Black RE, Cousens S, Dewey K, Giugliani E, et al. What works? Interventions for maternal and child undernutrition
and survival. Lancet. 2008:371(9610):417-440. doi:10.1016/S0140and survival. Lac effective complementary feeding behaviour change interventions
in developing countries. Matern Child Nutr. 2014;10(4):575-592.

16. Fabrizio CS, van Liere M, Pelto G. Identifying determinants of in developing countries.

17. Stewart RC, Umar E, Gleadow-Ware S, Creed F, Bristow K. Penatal distess and depression in Malawi: an exploratory qualitative study of stressors, supports and symptoms. Arch Womens Ment Health.

8. Roy SK, Jolly SP, Shafique S, Fuchs GJ, Mahmud Z, Chakraborty $\mathrm{B}$, et al. Prevention of malnutrition among young children in rural Bangladesh by a food-health-care educational intervention:
randomized, controlled trial. Food Nutr Bull. 2007;28(4):375-383 doi:10.1177/15648265070280040

19. Green J, Thorogood N. Qualitative Methods for Health Research. Second Edition. London: Sage; 2009 . 